\title{
Mathematical Modeling, Design and Development of Light-Weight Polyvinyl Chloride (PVC) Pipe Based Quadrotor for Monitoring an Outdoor Environment
}

\author{
Khan Muhammad, Naveed Sheikh", Abdul Rehman \\ Department of Mathematics, University of Balochistan, Quetta, Pakistan \\ Email address: \\ naveed_maths@hotmail.com (N. Sheikh) \\ ${ }^{*}$ Corresponding author \\ To cite this article: \\ Khan Muhammad, Naveed Sheikh, Abdul Rehman. Mathematical Modeling, Design and Development of Light-Weight Polyvinyl Chloride \\ (PVC) Pipe Based Quadrotor for Monitoring an Outdoor Environment. Mathematical Modelling and Applications. \\ Vol. 6, No. 1, 2021, pp. 10-16. doi: 10.11648/j.mma.20210601.12
}

Received: January 29, 2021; Accepted: March 24, 2021; Published: April 29, 2021

\begin{abstract}
The quadcopter, also known as an unmanned aerial vehicle (UAV), is a revolutionary innovation that has a great deal of potential. Modern quadrotors are transforming into small, powerful, light-weight, and agile vehicles. For the study, a variety of multirotor configurations were created before settling on a quadcopter structure. Our current focus is on mounting and designing a new polyvinyl chloride (PVC) pipe-based outdoor quadrotor setup. The quadcopter's controller is built based on the estimated mass output by decomposing the mathematical model, selecting the required motors, and using coherent electronic modules to track the outdoor environment. Quadrotor will take-off without generating any torque in the body casing. Rotors attached with motors generate thrust in the upward direction entirely based on the shape and dimension of the rotors. The proposed algorithm has strengthened the theory of nonlinear system output feedback control. The output control of a nonlinear system with parametric and functional uncertainties, as well as the input delay, is the most significant problem the configuration's materials were solely based on mass and forces acting on them. In this research we proposed a delayed roll and pitch angle reaction with standard specifications, and the faster roll and pitch angle reaction with appropriate parameters Quadrotor serves as the central body, tracking all controllable functions. Test results are then presented in order to show balanced flight performance.
\end{abstract}

Keywords: Quadrotor, Light-Weight, Unmanned Air Vehicle, Polyvinyl Chloride, Control

\section{Introduction}

The quadcopter is a drone that is lifted and powered by four propellers $[1,2]$. A quadcopter that produces propulsion dependent on four brushless motors and rotors [3, 4]. Two high-speed motors rotate in an anti-clockwise direction, while another two motors spin in the clockwise direction [5]. To stabilize the aircraft, these drones use an electronic control scheme and electronic sensors [6]. These moderate quadcopters can be operated outdoors along with their compact size and maneuverability; this form of configuration generates the torque from each motor to cancel by the opposing motor spinning in the reverse direction $[7,8]$. The main distinction with helicopter quadcopters is that the flyer uses adjustable thrust between the four brushless spinning engines to control pitch, yaw, and roll [9]. The shared lateral motions of a quadcopter are perfectly possible by precisely spinning these four brushless drone motors at varying speeds $[10,11]$.

The configuration proposed in this research paper consists of polyvinyl chloride (PVC) pipe based quadcopter by decomposition of the mathematical model to grab different surfaces (land or water). Quadrotor will take-off without generating any torque in the body casing. Rotors attached with motors generate thrust in the upward direction entirely based on the shape and dimension of the rotors. In this research Communication component is a transceiver that controls the quadcopter from ground station the quadcopter can be used for multipurpose applications like diagnostics, surveil-lance of unreachable places, finding missing people, etc. 


\section{Control Design and Working Principle for Quadrotor}

A quadrotor designed with cross beam four typical propeller structures in which two of them rotates at anticlockwise direction and others two rotate in clockwise $[12,13]$ direction as shown in Figure 1 (a), (b).
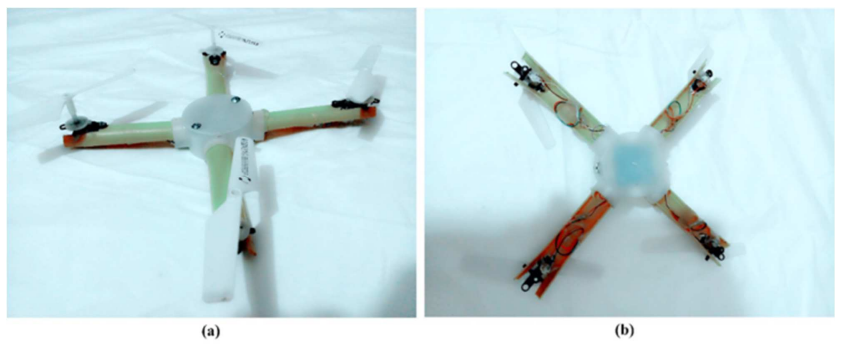

Figure 1. (a) Integrated Quadrotor. (b) upside down view of Quadrotor.

Quadrotor motion is attained by changing the rotation speed of the rotor discs, thus altering its torque load and thrust characteristics. The front and the rear rotors move counterclockwise, whereas the left and the right propeller rotate clockwise $[14,15]$. This form of inverse paired orientation configuration removes the need for a propeller, as shown in Figure 3. This arrangement illustrates the hovering modelling approach, where all the propellers have the same rpm. For individual rotor, two arrows are drawn: the curl arrow symbolizes the direction of rotation, the arrows show upward direction represents the thrust. These last vectors always point upwards so it may not chase the right hand rule since it likewise models an upright thrust [16, 17]. To accommodate for the gravitational acceleration, four rotors spin at the same speed. However, the quadrotor contains six DOF $[18,19]$. The quadrotor structure had four sophisticated controllable propellers which allow the quadrotor to attain a certain height and altitude [20,21].
A block diagram of the prototype description of the potential quadrotor as shown in Figure 2. The outputs are presented on the right side of block diagram, whereas the inputs are presented on the left.

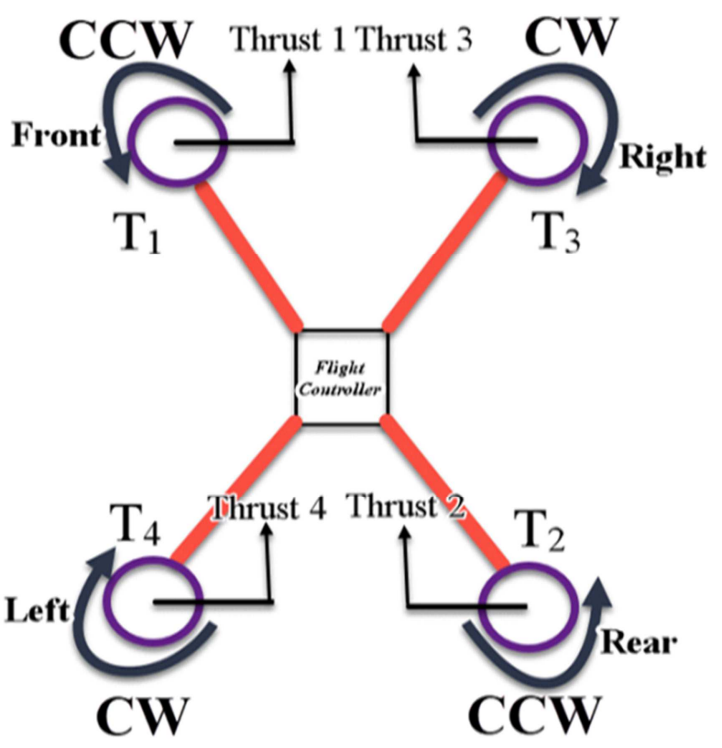

Figure 2. Block Diagram of Proposed Quadrotor.

With reference to the static reference frame, the quadrotor position can be computed by a translation vector with components $(\mathrm{x}, \mathrm{y}, \mathrm{z})$, orientation (pitch, roll, and yaw) and angles are $(\theta, \psi, \phi)$. A rotation by all of these 3 -angles of a rigid and stable body along Cartesian axes in 3-dimensional space could be accomplished by a rotation matrix $\mathrm{R}$ in the corresponding form as

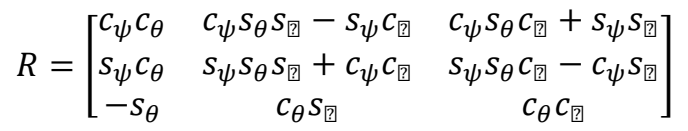

Since; $\operatorname{s} \alpha \equiv \sin \alpha, c \alpha \equiv \cos \alpha, \alpha=(\theta, \psi, \quad)$.

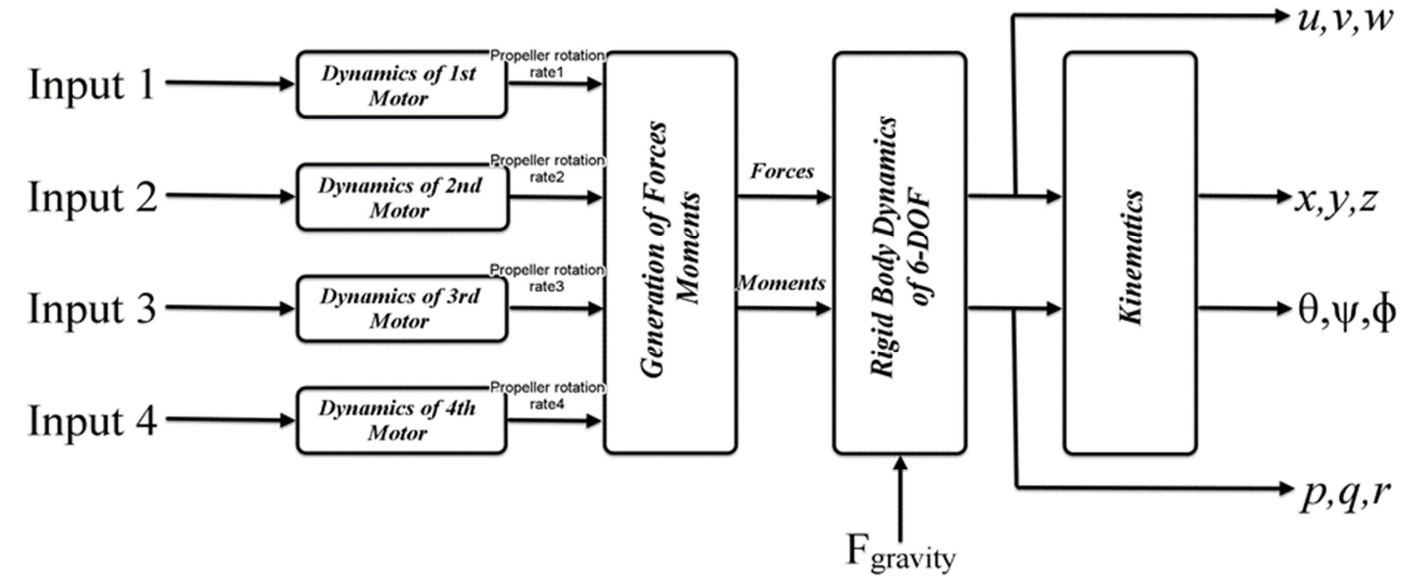

Figure 3. Quadcopter model schematic in hovering condition.

Consider a 3-dimensional schematic quadrotor model as shown in Figure 2, whereas (Thrust) $)_{i}, i=(1,2,3,4)$ is the Zcomponent of the ith rotor lift force vector (the additional components are expected to be ignored), $T_{i}$ is the speed of the rotor. Consider that the $3^{\text {rd }}$ and $4^{\text {th }}$ rotors rotate in clockwise direction and the $1^{\text {st }}$ and $2^{\text {nd }}$ rotate in counterclockwise direction. Also confirm that all the rotors rotate in the similar plane. 
This quadcopter configuration's output variable are 3linear coordinates $(\mathrm{x}, \mathrm{y}, \mathrm{z})$ in Cartesian space and 3-angles $(\theta$, $\psi, \quad$ ). Correspondingly, a system of 6-differential equations may be defined as the analogous model. Consider the quadcopter's corresponding dynamical model.

$$
\left\{\begin{array}{c}
m \ddot{x}=\left(\sum(\text { Thrust })_{i}\right)(\cos 0 \sin \theta \cos \psi+\sin 0 \sin \psi) \\
m \ddot{y}=\left(\sum(\text { Thrust })_{i}\right)(\sin 1 \sin \theta \cos \psi-\cos 1 \sin \psi) \\
m \ddot{z}=\left(\sum(\text { Thrust })_{i}\right)(\cos \theta \cos \psi)-m g \\
J_{1} \ddot{\theta}=l(\text { Thrust } 1-\text { Thrust } 2-\text { Thrust } 3+\text { Thrust } 4) \\
J_{2} \ddot{\psi}=l(- \text { Thrust } 1+\text { Thrust } 2-\text { Thrust } 3+\text { Thrust } 4) \\
J_{3} \ddot{0}=C(- \text { Thrust } 1-\text { Thrust } 2+\text { Thrust } 3+\text { Thrust } 4)
\end{array}\right.
$$

$\mathrm{J}_{1}, \mathrm{~J}_{2}, \mathrm{~J}_{3}$ are inertial moments of mass and $\mathrm{m}$ is the mass.

Applying the decomposition on independent model parameters with equivalent static nonlinear associations of a complex dynamic classical modeling. Consider introducing subsequent virtual power inputs which are superposition's of (Thrust)

$$
\left\{\begin{array}{c}
U_{1}=\text { Thrust } 1+\text { Thrust } 2+\text { Thrust } 3+\text { Thrust } 4 \\
U_{2}=\text { Thrust } 1-\text { Thrust } 2-\text { Thrust } 3+\text { Thrust } 4 \\
U_{3}=- \text { Thrust } 1+\text { Thrust } 2-\text { Thrust } 3+\text { Thrust } 4 \\
U_{4}=- \text { Thrust } 1-\text { Thrust } 2+\text { Thrust } 3+\text { Thrust } 4
\end{array}\right.
$$

Such virtual control responses will be determined on the basis of the coordinates indicated and the orientation of the potential quadcopter specified. The measurements of (Thrust) $)_{\mathrm{i}}$ will be calculated by the linear equation scheme given. By substituting the (3) in (4), we get the following simplified model result:

$$
\left\{\begin{array}{c}
m \ddot{x}=U_{1}(\cos 2 \sin \theta \cos \psi+\sin ? \sin \psi) \\
m \ddot{y}=U_{1}(\sin 2 \sin \theta \cos \psi-\cos ? \sin \psi) \\
m \ddot{z}=U_{1}(\cos \theta \cos \psi)-m g \\
J_{1} \ddot{\theta}=l U_{2} \\
J_{2} \ddot{\psi}=l U_{3} \\
J_{3} \ddot{?}=C U_{4}
\end{array}\right.
$$

Considering the $1^{\text {st }}$ two algebraic terms from the above described model (4). Matrix form can be written as

$$
\left[\begin{array}{c}
m \ddot{x} \\
m \ddot{y}
\end{array}\right]=\left[\begin{array}{cc}
\cos \theta & -\sin ? \\
\sin ? & \cos ?
\end{array}\right]\left[\begin{array}{c}
U_{1} \cos \psi \sin \theta \\
-U_{1} \sin \psi
\end{array}\right]
$$

where $\mathrm{U}_{1} \cos \psi \sin \theta$ and $-\mathrm{U}_{1} \sin \psi$ are auxiliary virtual control inputs

In this case, quadrotor control is necessary for the earthfixed coordinate system to be executed i.e. $(\mathrm{O}, \mathrm{X}, \mathrm{Y})$. However, we can suggest a supplementary coordination system $(\bar{O}, \bar{X}, \bar{Y})$ positioned correctly to the quadcopter for our convenience, as shown in Figure 4. Consider the displacement coordinates in the longitudinal directions of $\bar{x}$ and transverse directions of $\bar{y}$. Therefore, any transformation of the quadrotor can be specified in both the absolute and local coordinate system, as shown in Figure 4. Hence compose the relation between these system of coordinates

$$
\left[\begin{array}{l}
x^{\text {desired }} \\
y^{\text {desired }}
\end{array}\right]=\left[\begin{array}{l}
x \\
y
\end{array}\right]+\left[\begin{array}{cc}
\cos ? & -\sin ? \\
\sin ? & \cos ?
\end{array}\right]\left[\begin{array}{l}
\bar{x} \\
\bar{y}
\end{array}\right]
$$

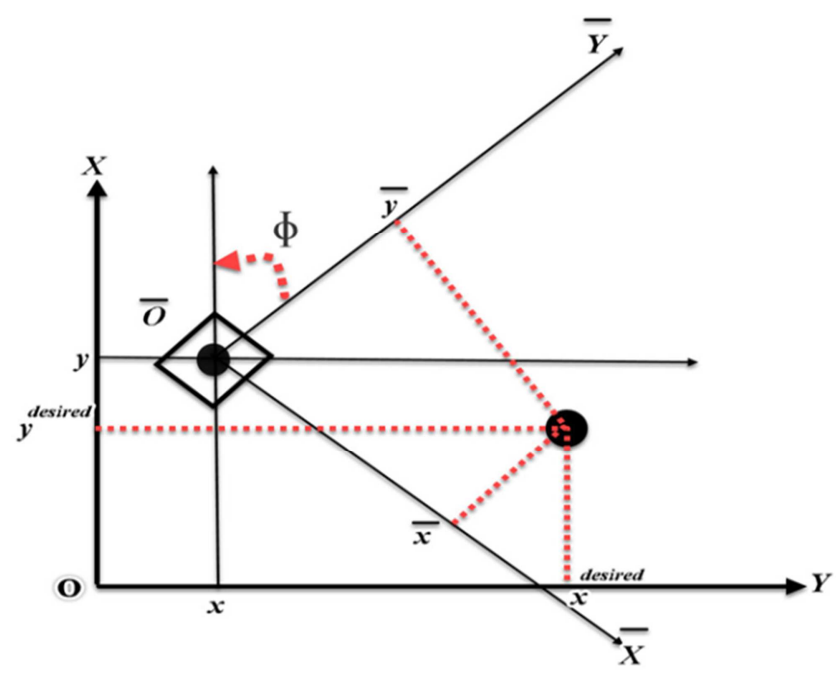

Figure 4. Co-ordinate system of the quadrotor.

Here $\left(x^{\text {desired }}, y^{\text {desired }}\right)$ and $(\mathrm{x}, \mathrm{y})$ the desired and actual quadrotor coordinate system are in the absolute coordinate system, respectively. Thus, for planar coordinate systems, the matrix is a rotation matrix. Represent the above algebraic equation (6) in the form of system coordinates $\bar{x}$ and $\bar{y}$ we get the following equation.

$$
\left[\begin{array}{l}
\bar{x} \\
\bar{y}
\end{array}\right]=\left[\begin{array}{cc}
\cos ? & \sin ? \\
-\sin ? & \cos ?
\end{array}\right]\left[\begin{array}{l}
x^{\text {desired }}-x \\
y^{\text {desired }}-y
\end{array}\right]
$$

Double Differentiate and multiply ' $m$ ' to equation (7). We have

$$
\left[\begin{array}{c}
\ddot{\bar{x}} \\
\ddot{\bar{y}}
\end{array}\right]=\left[\begin{array}{cc}
\cos ? & \sin ? \\
-\sin ? & \cos ?
\end{array}\right]\left[\begin{array}{l}
-m \ddot{x} \\
-m \ddot{y}
\end{array}\right]
$$

Substitute equation (5) in equation (8) and develop the transformation model.

$$
\left[\begin{array}{c}
m \ddot{\bar{x}} \\
m \ddot{\bar{y}}
\end{array}\right]=\left[\begin{array}{c}
-\left(U_{1} \cos \psi \sin \theta\right) \\
-\left(-U_{1} \sin \psi\right)
\end{array}\right]
$$

Changing roll or pitch angles is clearly a helping technique to validate a change in the coordinate system $\bar{x}$ and $\bar{y}$. Changes of these critical values are permitted within a minute span., for example, $\left[\frac{-\pi}{6}, \frac{\pi}{6}\right]$. It is reasonable in this situation to use the predicted equations $\sin \alpha \approx \alpha, \cos \alpha \approx 1$ if $|\alpha| \leq \frac{\pi}{6}$.

Reconfigure $3^{\text {rd }}$ equation from (4) and auxiliary virtual control inputs $U_{1} \cos \psi \sin \theta,-U_{1} \sin \psi$ in the following form

$$
\begin{gathered}
\mathrm{m} \ddot{z}=U_{1}-m g \\
U_{1} \cos \psi \sin \theta=U_{1} \theta=U_{5}(\operatorname{say}) \\
-U_{1} \sin \psi=-U_{1} \psi=U_{6}(\operatorname{say})
\end{gathered}
$$

So that we can compose the much more improved and simpler quadrotor model 


$$
\left\{\begin{array}{c}
m \ddot{\bar{x}}=-U_{5} \\
m \ddot{\ddot{y}}=-U_{6} \\
m \ddot{z}=U_{1}-m g \\
J_{1} \ddot{\theta}=l U_{2} \\
J_{2} \ddot{\psi}=l U_{3} \\
J_{3} \ddot{\ddot{z}}=C U_{4}
\end{array}\right.
$$

where the system of local coordinates $\bar{x}$ and $\bar{y}$ can be calculated by equation (7) in conjunction with the necessary system of co-ordinates in relation to the absolute system of co-ordinates, the desired parameters of $\theta$ and $\psi$ could be taken out from equation (11) and (12)

$$
\left\{\begin{array}{l}
\theta^{\text {desired }}=\frac{U_{5}}{U_{1}} \\
\psi^{\text {desired }}=\frac{-U_{6}}{U_{1}}
\end{array}\right.
$$

So here we can conclude that if $U_{1}$ is approaching to zero then the values of $\theta^{\text {desired }}$ and $\psi^{\text {desired }}$ need to be equivalent to 0 . It specifies that the motion is more optimal along the $\mathrm{z}$ coordinate directed by $\mathrm{U}_{1}$. If the absolute value of $U_{1}$ is greater than or equal, one may use the equation (14) of any predetermined constant. In addition, if the result of (14) is too high, the required pitch or roll is larger than the critical value such as $\frac{\pi}{6}$, it is efficient to attach any predetermined constant to the desired values of $\theta^{\text {desired }}$ or $\psi^{\text {desired }}$.

Here It is simple to deal with the output control method to establish the equivalent virtual controllers for $U_{i}$ where $\mathrm{i}=$ $1 \rightarrow 6$ with $\rho=2$.

$$
\begin{gathered}
U_{1}=m g+\mu_{1}\left(\xi_{1}+\dot{\xi}_{1}\right), \dot{\xi}_{1}=\sigma_{1}\left(-\xi_{1}+z^{\text {desired }}-z\right) \\
U_{2}=\mu_{2}\left(\xi_{2}+\dot{\xi}_{2}\right), \dot{\xi}_{2}=\sigma_{2}\left(-\xi_{2}+\theta^{\text {desired }}-\theta\right) \\
U_{3}=\mu_{3}\left(\xi_{3}+\dot{\xi}_{3}\right), \dot{\xi}_{3}=\sigma_{3}\left(-\xi_{3}+\psi^{\text {desired }}-\psi\right) \\
U_{4}=\mu_{4}\left(\xi_{4}+\dot{\xi}_{4}\right), \dot{\xi}_{4}=\sigma_{4}\left(-\xi_{4}+\text { ? } \text { desired }- \text { ? }\right) \\
U_{5}=\mu_{5}\left(\xi_{5}+\dot{\xi}_{5}\right), \dot{\xi}_{5}=\sigma_{5}\left(-\xi_{5}+\bar{x}\right) \\
U_{6}=\mu_{6}\left(\xi_{6}+\dot{\xi}_{6}\right), \dot{\xi}_{6}=\sigma_{6}\left(-\xi_{6}+\bar{y}\right)
\end{gathered}
$$

$\bar{x}$ and $\bar{y}$ can be obtained by equation (7). So we will develop completely the virtual control inputs $U_{i}$. We are implementing the inverse transformation of static nonlinearity to identify equations for lift forces generated by actuators. In the linear equation (3), substitute the first four control inputs, $\mathrm{U}_{1}, \mathrm{U}_{2}, \mathrm{U}_{3}$, and $\mathrm{U}_{4}$. So we get the values of Thrust1, Thrust2, Thrust 3 and Thrust 4 for the lift forces.

\section{Simulation}

We have quadrotor parameters $\mathrm{m}, \mathrm{l}, \mathrm{C}, \mathrm{J}_{1}, \mathrm{~J}_{2}, \mathrm{~J}_{3}$ and $\mathrm{g}$. The initial co-ordinates $\left(\mathrm{x}_{0}, \mathrm{y}_{0}, \mathrm{z}_{0}\right)=(0,0,0)$ and the initial orientation is $\phi_{0}=0$. We have reference points $\left(\mathrm{x}^{\text {desired }}, \mathrm{y}^{\text {desired }}\right.$, $\left.\mathrm{z}^{\text {desired }}\right)$ and the desired orientation is $\phi^{\text {desired }}$. We have coefficients $\mu_{1}, \mu_{2}, \mu_{3}, \mu_{4}, \mu_{5}, \mu_{6}, \sigma_{1}, \sigma_{2}, \sigma_{3}, \sigma_{4}, \sigma_{5}, \sigma_{6}$.

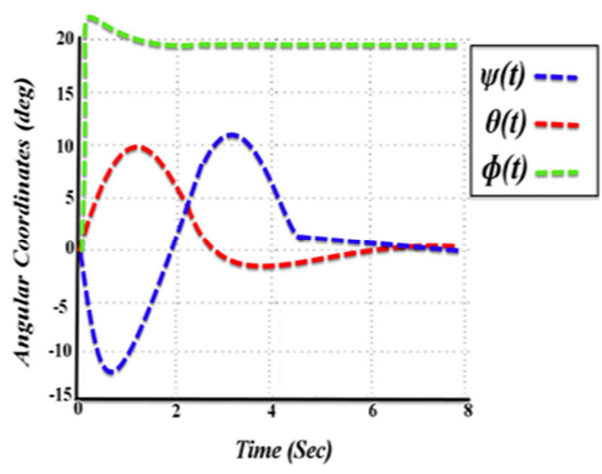

Figure 5. Transients for Angular co-ordinates.

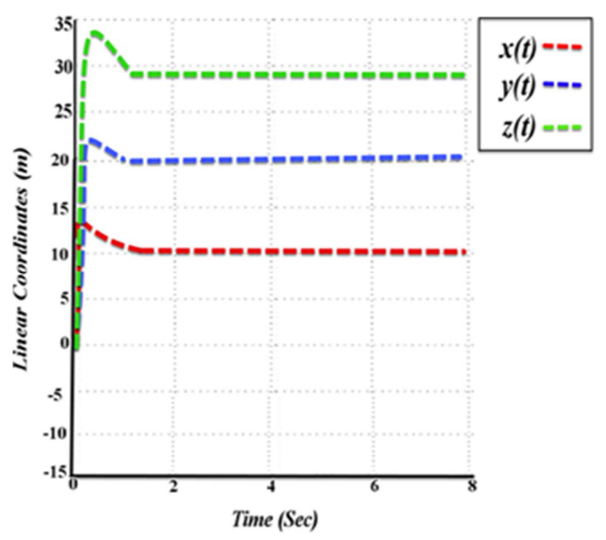

Figure 6. Transients for Linear co-ordinates.

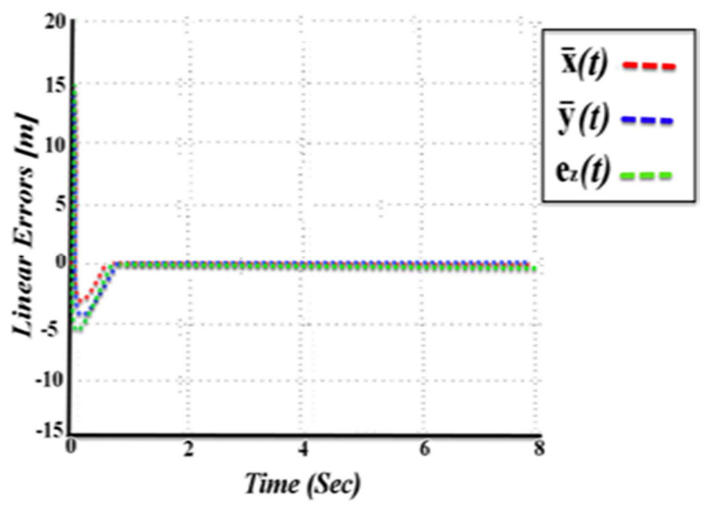

Figure 7. Transients for linear errors.

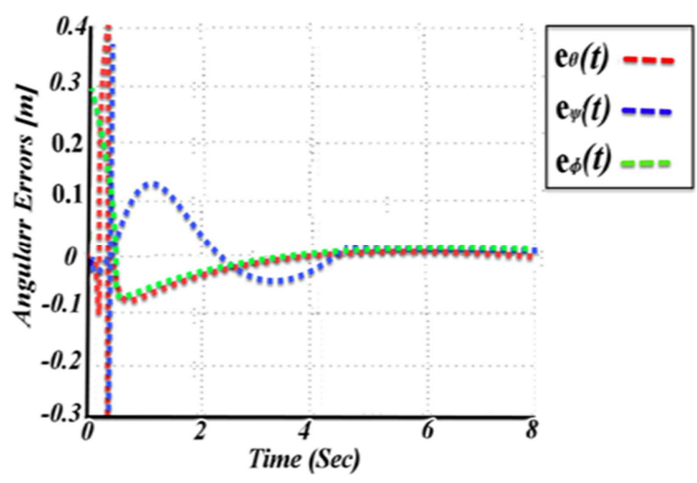

Figure 8. Transients for angular errors.

Figures 5 and 6 explain the transients for angular coordinates and transients for linear co-ordinates respectively 
of the closed loop system. whereas Figures 7 and 8 illustrate the transients for the linear errors and transients for angular errors respectively.

\section{Experimental Setup and Results}

As shown in Figure 1, plastic pipes for the whole body were used to minimize the weight of the platform. Furthermore, we have tried to keep the quadrotor as compact as feasible so that in outdoor atmosphere it operates effectively. In Table 1, the lighter overall mass of the integrated battery-powered quad-rotor, which is approximately one tenth of the commercial quad-rotor, ensures that it can be an energy-efficient device relative to the commercial quadrotor. In addition, the smaller scale, suggests that using it in an outdoor environment would be beneficial

Table 1. Parameters of proposed quadrotor.

\begin{tabular}{ll}
\hline Parameters & Designed Quadrotor \\
\hline Height & $56 \mathrm{~mm}$ \\
Diameter & $210 \mathrm{~mm}$ \\
Bladed & $3 "$ \\
Weight & $34 \mathrm{~g}$ \\
\hline
\end{tabular}

Table 2. Parameters values used in PD controllers.

\begin{tabular}{llll}
\hline Parameters & Values & Parameters & Values \\
\hline $\mathrm{K}_{\mathrm{p} 1}$ & 0.30 & $\mathrm{~K}_{\mathrm{d} 1}$ & 2.10 \\
$\mathrm{~K}_{\mathrm{p} 2}$ & 5 & $\mathrm{~K}_{\mathrm{d} 2}$ & 0.6 \\
$\mathrm{~K}_{\mathrm{p} 3}$ & 15 & $\mathrm{~K}_{\mathrm{d} 3}$ & 4.09 \\
$\mathrm{~K}_{\mathrm{p} 4}$ & 1 & $\mathrm{~K}_{\mathrm{d} 4}$ & 0.55 \\
\hline
\end{tabular}

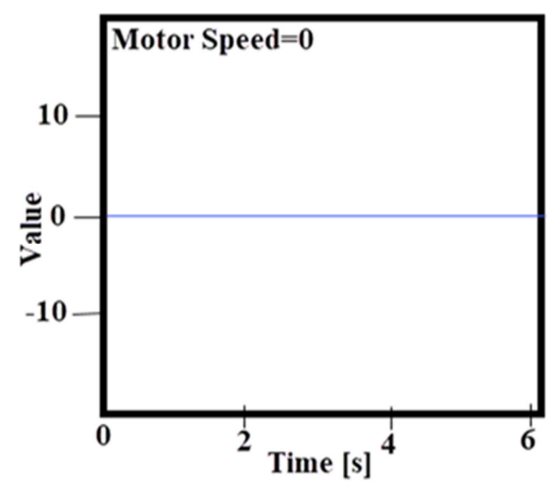

Figure 9. Evaluated noisy sensor data while motor s speed is zero.

Figures 9 and 10 illustrates that the angular information from the accelerometer sensor is calculated, which is linked on the circuit board, as accelerating generators, they are coupled with noise. We developed the RC filter to remove the noise resulting from body movements. It consists of multiple resistors and capacitors. The efficiency of the control system suggested is shown in Figure 11 to 16. Figure 11, 12, 13 and 14 indicates the PD controller's reaction to the stabilization of the quadrotor from the flipped body at 400. Furthermore, during tests, we did not avoid any disruptions such as air because we believed that the designed UAV was used outdoor environment. Table 2 provides the constraints that were used with PD controllers. But many parameters were extracted from tuning gains before a better device performance

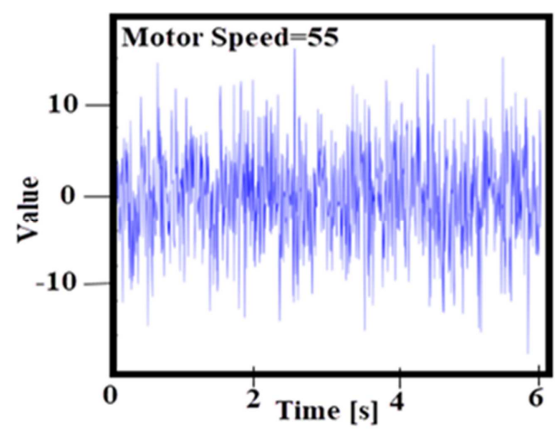

Figure 10. Evaluated noisy sensor data while motor s speed is 55 .

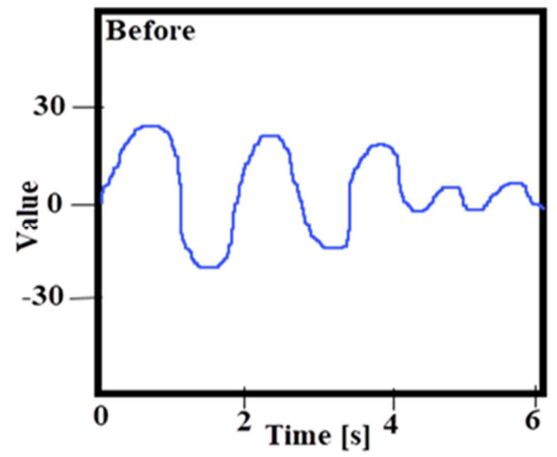

Figure 11. Roll angles modifications with PD regulation (Before).

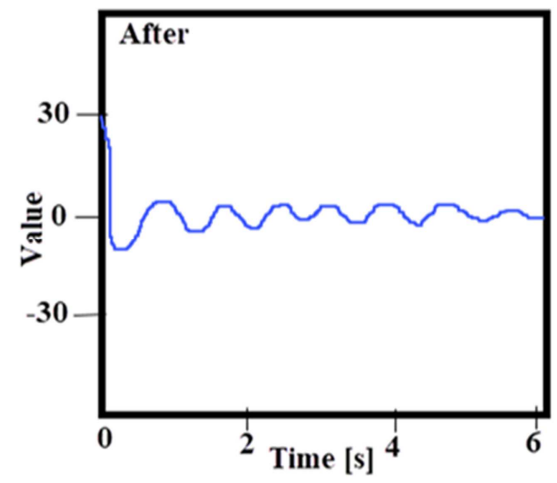

Figure 12. Roll angles modifications with PD regulation (After).

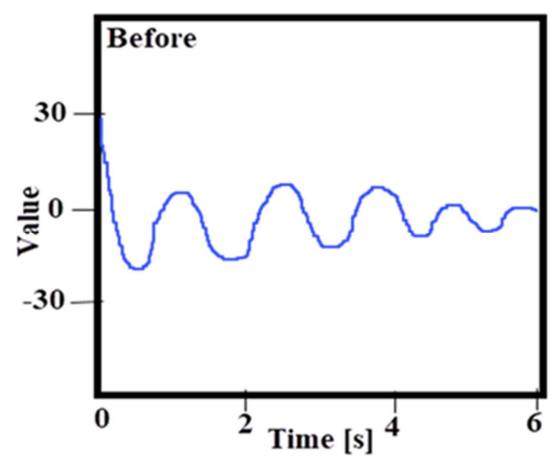

Figure 13. Pitch angles modifications with PD regulation (Before). 


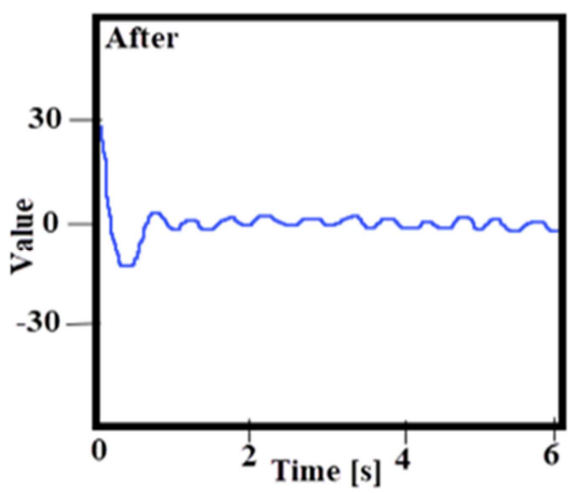

Figure 14. Pitch angles modifications with PD regulation (After).

In Figures 11 and 13, illustrate the delayed roll and pitch angle reaction with standard specifications, and the Figure 12 and 14 display the faster roll and pitch angle reaction with appropriate parameters. Therefore, Figure 9 illustrates the angles of roll and pitch while hovering outdoors. Consequently, the micro quadrotor UAV rotational framework appears to be entirely manageable and measurable. Ultimately, we checked the efficiency of its success in the actual outdoor conditions.

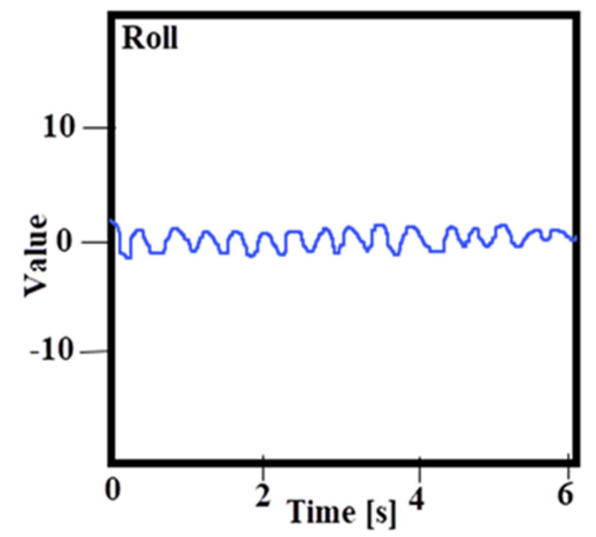

Figure 15. Observational quad-rotor outputs at hover (Roll).

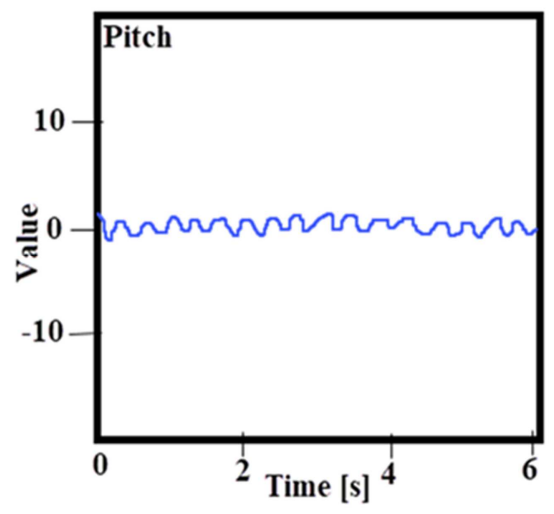

Figure 16. Observational quad-rotor outputs at hover (Pitch).

\section{Conclusion and Future Work}

The recent advancements in the development of the output control approach are the subject of this paper. The proposed algorithm has strengthened the theory of nonlinear system output feedback control. The output control of a nonlinear system with parametric and functional uncertainties, as well as the input delay, is the most significant problem. In previous research, the control issue for linear plants with input delays was considered. The feedback controller, which is based on methods outlined in several research papers, allows an internally unstable plant with the input delay to reject an unknown biased sinusoidal disturbance. In this article, therefore, we have proposed the light-weight polyvinyl chloride (PVC) pipe based quadrotor and the optimized stabilization control algorithm were developed. In addition, it was incorporated at reasonable cost, under the basis that the specifications are exceptional. As a consequence, the experimental findings have shown that, in terms of stable flight, the constructed platform performs efficiently.

\section{References}

[1] C. Coza, C. Nicol, C. J. B. Macnab, and A. Ramirez-Serrano, "Adaptive fuzzy control for a quadrotor helicopter robust to wind buffeting," J. Intell. Fuzzy Syst., vol. 22, pp. 267-283, 2011.

[2] Y. Tao et al., "A PID and fuzzy logic based method for Quadrotor aircraft control motion," J. Intell. Fuzzy Syst., vol. 31, no. 6, pp. 2975-2983, 2016.

[3] J. Estevez, M. Graña, and J. M. Lopez-Guede, "Online fuzzy modulated adaptive PD control for cooperative aerial transportation of deformable linear objects," Integr. Comput. Aided. Eng., vol. 24, no. 1, pp. 41-55, 2017.

[4] K. Choutri, L. Mohand, and L. Dala, "Design of search and rescue system using autonomous Multi-UAVs," vol. 1, pp. 1$12,2020$.

[5] K. M. Zemalache, L. Beji, and H. Maaref, "Two inertial models of X4-flyers dynamics, motion planning and control," Integr. Comput. Aided. Eng., vol. 14, no. 2, pp. 107-119, 2007.

[6] L. A. Paramo et al., "Quadrotor stabilization by Fuzzy Kalman Filter,” J. Intell. Fuzzy Syst., vol. 38, pp. 4485-4494, 2020.

[7] S. Id and W. Count, "Modified WingsFrame Design of a Low Cost Unmanned Aerial Vehicle (UAV) Quad Rotor by using the PlasticRubber Air Tube for Vertical Takeoff and Landing on Water \& Dry," 2017.

[8] A. A. Pyrkin, A. A. Bobtsov, S. A. Kolyubin, O. I. Borisov, and V. S. Gromov, "Output controller for quadcopters based on mathematical model decomposition," 2014 22nd Mediterr. Conf. Control Autom. MED 2014, pp. 1281-1286, 2014.

[9] G. S. B. Shaik Himam Saheb, "Design and Analysis of Light Weight Agriculture Robot," Glob. J. Res. Eng., vol. 17, no. 6, 2017.

[10] M. Sudarma, I. B. Alit Swamardika, and A. Mas Pratama, "Design of quadcopter robot as a disaster environment remote monitor," Int. J. Electr. Comput. Eng., vol. 6, no. 1, pp. 188197, 2016. 
[11] A. SS and M. R, "Design and Fabrication of Voice Controlled Unmanned Aerial Vehicle," J. Aeronaut. Aerosp. Eng., vol. 5, no. 2,2016

[12] S. K. Phang, C. Cai, B. M. Chen, and T. H. Lee, "Design and Mathematical Modeling of a 4-Standard-Propeller.pdf," pp. $3270-3275$.

[13] G. Ostojić, S. Stankovski, B. Tejić, N. Dukić, and S. Tegeltija, "Design, control and application of quadcopter," Int. J. Ind. Eng. Manag., vol. 6, no. 1, pp. 43-48, 2015.

[14] D. B B V L and P. Singh, "A survey on design and development of an unmanned aerial vehicle (quadcopter)," Int. J. Intell. Unmanned Syst., vol. 4, no. 2, pp. 70-106, 2016.

[15] S. Badr, O. Mehrez, and A. E. Kabeel, "A novel modification for a quadrotor design," in 2016 International Conference on Unmanned Aircraft Systems, ICUAS 2016, 2016.

[16] D. Fitzgerald, R. Walker, and D. Campbell, "A Vision Based Forced Landing Site Selection System for an Autonomous UAV," 2005 Int. Conf. Intell. Sensors, Sens. Networks Inf. Process., pp. 397-402, 2005.
[17] S. K. Phang, K. Li, K. H. Yu, B. M. Chen, and T. H. Lee, "Systematic Design and Implementation of a Micro Unmanned Quadrotor System," vol. 2, no. 2, pp. 121-141, 2014.

[18] T. T. H. Ng and G. S. B. Leng, "Design of small-scale quadrotor unmanned air vehicles," vol. 221, pp. 893-906, 2007.

[19] V. Lippiello, F. Ruggiero, and D. Serra, "Emergency landing for a quadrotor in case of a propeller failure: A backstepping approach," IEEE Int. Conf. Intell. Robot. Syst., no. Iros, pp. 4782-4788, 2014.

[20] O. Dunkley, J. Engel, J. Sturm, and D. Cremers, "VisualInertial Navigation for a Camera-Equipped $25 \mathrm{~g}$ NanoQuadrotor," IROS2014 Aer. Open Source Robot. Work., pp. 4 5, 2014.

[21] J. B. Devaud, S. Najko, P. Le Nah??dic, C. Maussire, E. Zante, and J. Marzat, "Full design of a low-cost quadrotor UAV by student team," in Proceedings of the 2012 International Conference on System Engineering and Technology, ICSET 2012. 